\title{
On the integration of Cid's radial intermediary
}

\author{
Alberto Abad ${ }^{1}$ \\ Universidad de Zaragoza, 50009 Zaragoza, Spain. \\ Manuel Calvo ${ }^{1}$ \\ Universidad de Zaragoza, 50009 Zaragoza, Spain. \\ Antonio Elipe ${ }^{1, *}$ \\ Universidad de Zaragoza, 50009 Zaragoza, Spain.
}

\begin{abstract}
This paper deals with the integrations of homogeneous quasi-Keplerian Hamiltonians, that is, perturbed Kepler Hamiltonians which perturbation is of the form $\sum_{j=2}^{N} A_{j} / r^{j}$ with $A_{j}$ constant. Although there are many applications of these Hamiltonians in Physics, Astronomy and Astrodynamics, we focus our interest on a particular case in the core of Artificial Satellite Theory, the Cid's radial intermediary. For this problem, we integrate the equations of motion in two different ways, by means of the elliptic $\mathcal{P}$-Weierstrass function and by using the Krilov-Bogoliubov averaging method to integrate a perturbed harmonic oscillator. In this case, the resulting solution is given in terms of the classical Kepler's equation, with no need of introducing more complex generalized Kepler's equation.

Keywords: Artificial Satellite Theory, Quasi-Keplerian

Hamiltonians, Radial Intermediaries, Kepler's Equation
\end{abstract}

\footnotetext{
* Corresponding author

Email address: elipe@unizar.es (Antonio Elipe)

${ }^{1}$ Instituto Universitario de Matemáticas y Aplicaciones.
} 


\section{Introduction.}

Hamiltonians of the type

$$
\mathcal{H}=\sum_{j=0}^{N} \mathcal{H} j=\mathcal{H}_{K}+\sum_{j=2}^{N} \frac{A_{j}}{r^{j}}, \quad\left(A_{j} \text { constant }\right)
$$

are known as quasi-Keplerian Hamiltonians since they are extensions of the Kepler problem $\left(\mathcal{H}_{K}\right)$.

The first case we find in the literature was given by Newton in Liber II, 5 Propositio XLIV of his Philosophiae Naturalis Principia Mathematica ([27]). Indeed, a short sentence at the end of Corollarium 2 of Propositio XLV reveals that Newton considered to explain departures in the orbit of the Moon from a mere Keplerian orbit as the effect of an additional central repulsion. The result is that the motion was not a pure ellipse but a precessing ellipse, originating a rosette-like orbit.

Manev potential [23, 24]

$$
U=-\mathcal{G} \frac{m_{1} m_{2}}{r}\left(1+\frac{3 \mathcal{G}\left(m_{1}+m_{2}\right)}{2 c^{2} r}\right),
$$

with $\mathcal{G}$ the Gaussian constant, $m_{1}$ and $m_{2}$ the masses of the two particles and $c$ light's speed was introduced to describe the secular motions of Mercury's perihelium. Though simple, this work appeared at the same period as Einstein's General Relativity and no much attention was paid to it at that time. The use of Manev-like potential

$$
U=-\frac{A}{r}-\frac{B}{r^{2}}
$$

was reintroduced by Hagihara [17 and since then, many papers dealing with Manev-like potentials have appeared [25, 21, 5, 6, 7.

Potentials of Schwarzschild-type [26] also belong to quasi-Keplerian potentials. They are of the form

$$
U=-\frac{A}{r}-\frac{B}{r^{3}},
$$

With $A$, and $B$ non negative constants. Although it has been extensively used in problems related with black holes, it has also many applications in other fiels, 
Another example of quasi-Keplerian problem is the Fock's potential, that may be put in the form

$$
U=-\frac{\mu}{r}\left(1+\sum_{j=0}^{3} \frac{A_{j}}{r^{j}}\right),
$$

made of the Keplerian part plus the relativistic perturbation.

In Artificial Satellite Theory (AST) we find potentials of the above types when some Lie-transforms are applied to the main-problem, that is, when only the influence of the oblateness (the second zonal harmonic term in the expansion of the potential) is considered. They are what Deprit [13] dubbed radial intermediaries. We will mention later on the ones obtainend by Deprit [13] (of Manev's type) and Cid [12] (of Schwazrschild's type). This type of Hamiltonians was one of the main goals of Deprit philosophy, how to obtain (helped by mathematics and computing science) simpler Hamiltonians containing most of 25 the dynamics of the original Hamiltonian, which in Deprit's words was Simplify or Perish [14. There are many examples in AST were these intermediaries have been used. Let us mention for instance a recent work of Lara and Gurfil [20] where they used Cid's intermediary in a formation flihgt problem in which two spacecraft describe neighbouring orbits with the same period so that they remain close for long time intervals. To simplify the study of this problem the above mentioned authors have considered Cid's intermediary instead of the general satellite equations.

The main characteristic of problems with quasi-Keplerian potentials is that they are integrable, since they are problems of one-degree of freedom in $r$. However, the solution usually is not easy to find, since in most cases, it is given in terms of rather involved expressions of elliptic functions, and from the practical point of view not very useful due to the high computational cost. Hence although for low powers in the potential it is feasible to have the solution directly by quadrature, in practice, alternative solutions are sought. Even for the 40 classical Kepler problem, which solution does not involve elliptic functions but circular ones for negative energies, a direct integration to obtain $r=r(t)$ from 
the first integral of energy does not provide $r$ as a function of time that is the main interest but the inverse function, $t$ as a function of $r$. To get explicit expressions of $r$ in terms of time $t$, this is usually achieved by the anomalies (true 45 or eccentric ones) and their relation with the independent variable is expressed through Kepler's equation.

For more general potentials like (3) some authors [20] have studied the integration of the energy integral with $B \ll A$ obtaining the time in terms of an integral of a cubic quadrature that also requires the introduction of elliptic integrals.

One way of finding the solution of Hamiltonian (1) is by regularization and linearization techniques, which, essentially consist of a change of the independent variable $(t \mapsto \tau)$ by the relation $d t=g(r) d \tau$ in order the equation of motion be reduced to a harmonic oscillator. There are two difficulties: Firstly to ensure the existence and practical calculation of $g(r)$, and secondly to obtain the explicit relation between $t$ and $\tau$ from

$$
\frac{d \tau}{d t}=\frac{1}{g(r(t))} .
$$

For instance, Cid, Ferrer and Elipe [10] for the potential

$$
U=\sum_{j=1}^{n} \frac{a_{j}}{r^{j}},
$$

proposed the regularizing function $g_{n}(r)=r^{n / 2}\left(r^{n-2}+\alpha_{1} r^{n-3}+\cdots+\alpha_{n-2}\right)^{-1 / 2}$ and the equation of motion is reduced to

$$
\frac{d^{2} r}{d \tau}=2 c_{1} r+c_{2}
$$

which corresponds to a harmonic oscillator. Note that $\alpha_{j}, c_{1}$ and $c_{2}$ are defined by a set of non linear equations and hence, it is not clear the existence of a real solution. However, for $\alpha_{1}=1$ and all remaining $\alpha_{j}$ sufficiently small, by application of the theorem of implicit functions there exists a real solution for ${ }_{55} \alpha_{j}, c_{1}$ and $c_{2}$.

Similar linearizing function was given by Ferrándiz [15] and obtained the expression of the independent variable $(t)$ in terms of elliptic functions. 
In a recent paper, López, Hautesserres and San-Juan [22] dealt with first order of perturbation Deprit's intermediary (we recall, it is of the type (2p), and after an appropriate change of variables, they end up with a perturbed harmonic oscillator. To it, they applied the Krylov-Bogoliubov-Mitropolski perturbation method and obtained the independent variable $(t)$ by means of a generalized Kepler's equation, in the same way as it was done in 3] for the second order of perturbation Deprit's intermediary. More recently, the authors [1, with a similar technique to the one used in this paper, were able to provide the solution of Deprit's intermediary, and determined the physical time $t$ by means of the classical Kepler's equation, which solution has been well studied along the years, with no need of introducing a generalized Kepler's equation. This last work motivated the present paper, the obtaining of a solution of Cid's intermediary by making use of the classical Kepler's equation instead of a generalizad Kepler's equation.

The paper is organized as follows: In the next Section we summarize the two most used intermediaries, namely Deprit's and Cid's ones in such a way that both intermediaries are presented in a unique formula. Since Deprit's intermediary was integrated elsewhere [1, we focus our interest only in Cid's intermediary. The integration is carried out in Section 3. By means of an appropriate change of variables, including the independent variable, we are able to put the equations of motion in the form of a perturbed harmonic oscillator. Note at this point that we do not use a classical function linearization transformation like Sundman's one 28, but two transformations, one changing the radial distance by its inverse and a second one changing the physical time by the polar angle. Then, we proceed to the integration, firstly in terms of elliptic functions, in particular the $\mathcal{P}$-Weierstrass funcion (Subsection 3.1), and secondly, by means of the KB-averaging method [18] (Subsection 3.2). For this averaged solution, we 85 manage to relate the time and a elliptic-type anomaly by a Kepler's equation, which has been well studied for more than three centuries. It is worth to note that the KB-averaging method provides some bounds to the obtained solution, which makes it very convenient for the long term integration. 


\section{Radial intermediaries in AST}

In the Main Problem of the artificial satellite that describes the motion of a satellite around an Earth-like planet taking into account only the $J_{2}$ effect (planet's oblateness) considered a perturbation to the two-body problem. The use of first order perturbation theories in the case of elliptic type orbits leads to a perturbation of Kepler's equation that must be solved to define the position of the satellite at each required time. The way to reach the perturbed harmonic oscillator is not unique; it depends on the type of variables used and also on the different transformations employed to eliminate some variables which leads to what is called intermediaries [13.

The Hamiltonian of the main problem in Whittaker (also known as polarnodal) variables $(r, \theta, \nu, R, \Theta, N)$ is

$$
\mathcal{H}_{m}=\frac{1}{2}\left(R^{2}+\frac{\Theta^{2}}{r^{2}}\right)-\frac{\mu}{r}+\epsilon \frac{\mu}{r}\left(\frac{\alpha}{r}\right)^{2} P_{2}(\sin I \sin \theta),
$$

where $\epsilon=J_{2}$ is considered a small parameter, $\alpha$ the equatorial radius of the planet, $I$ the inclination $(\cos I=N / \Theta), \mu$ the gravitational parameter, and $P_{2}(x)$. the Legendre polynomial of second degree. Note that we only consider $\Theta>0$, hence rectilinear orbits are excluded.

An intermediary is a simplification, in the sense that it is made of an integrable Hamiltonian, the dominant part, whereas the remainder is a perturbation affecting the intermediary. For details see the seminal Deprit's paper [13] or the most recent article by Lara [19]. The two radial intermediaries most used are those named after Deprit and Cid. Let us schematically see how to obtain them.

Deprit's intermediary. It is obtained after the application of two Lie tranforms to the Hamiltonian (5), namely the elimination of the parallax [13] and the elimination of the perigee [4. The resulting Hamiltonian is

$$
\mathcal{H}_{d}(r, R)=\frac{1}{2}\left(R^{2}+\frac{\Theta^{2}}{r^{2}}\right)-\frac{\mu}{r}+\epsilon \frac{\Phi_{\mathcal{D}}}{r^{2}},
$$

with

$$
\Phi_{\mathcal{D}}=\Theta^{2}\left(\frac{\alpha}{p}\right)^{2}\left(\frac{1}{2}-\frac{3}{4} \sin ^{2} I\right)=\frac{\alpha^{2} \mu^{2}}{\Theta^{2}}\left(\frac{1}{2}-\frac{3}{4} \sin ^{2} I\right)
$$


and $p$ is the semi-latus rectum.

Cid's intermediary. The two Lie tranforms to be applied to Hamiltonian (5), are firstly the elimination of the latitude $(\theta)$ and then, the elimination of the perigee. The result is the Hamiltonian

$$
\mathcal{H}_{c}(r, R)=\frac{1}{2}\left(R^{2}+\frac{\Theta^{2}}{r^{2}}\right)-\frac{\mu}{r}+\epsilon \frac{\Phi_{\mathcal{C}}}{r^{3}}, \quad \text { with } \quad \Phi_{\mathcal{C}}=-\mu \alpha^{2}\left(\frac{1}{2}-\frac{3}{4} \sin ^{2} I\right) .
$$

For details, the reader is addressed to [12, 11] and 9. Note that when comparing $\Phi_{\mathcal{C}}$ with he expression $H_{0,1}$ of $[9$, p. 245] or with [11, Eq. (12)], there is a difference on the sign, due that in our work we take $\epsilon=J_{2}$, but in the mentioned papers they took $\epsilon=-J_{2}$. Besides, in those papers, $\alpha=1$ since it was taken as the length unit.

In both cases the two angular variables, $(\theta, \nu)$, have been eliminated from the Hamiltonian (5), and therefore their conjugate momenta, $(\Theta, H)$, are constant and the intermediaries only depend on $(r, R)$, hence the name of radial intermediaries, which we may write in a unique formula as

$$
\mathcal{H}_{r}(r, R)=\frac{1}{2}\left(R^{2}+\frac{\Theta^{2}}{r^{2}}\right)-\frac{\mu}{r}+\epsilon\left(\frac{\Phi_{\mathcal{D}}}{r^{2}}+\frac{\Phi_{\mathcal{C}}}{r^{3}}\right),
$$

When $\Phi_{\mathcal{C}}=0$ and $\Phi_{\mathcal{D}}$ as in Eq. (6) we have Deprit's intermediary. When $\Phi_{\mathcal{D}}=0$ and $\Phi_{\mathcal{C}}$ as in Eq. (7) we have Cid's one. Note also that $\Phi_{\mathcal{D}}$ and $\Phi_{\mathcal{C}}$ are constant.

The integration of Deprit's radial intermediary has been solved in [1]. By means of some appropriate change of variables, including the independent variable $t$, the problem is reduced to a Keplerian problem. Integration of Cid's intermediary case is more involved and two different ways of obtaining it will 125 be presented in the next sections. 


\section{Integration of Cid's radial intermediary}

Hamilton's equations corresponding to the one-dimensional Hamiltonian (7) can be reduced to the second order differential equation in $r=r(t)$

$$
\frac{d^{2} r}{d t^{2}}=\frac{\Theta^{2}}{r^{3}}-\frac{\mu}{r^{2}}+3 \varepsilon \frac{\Phi}{r^{4}} .
$$

where we put $\Phi=\Phi_{\mathcal{C}}$ since we are dealing only with Cid's intermediary.

Note that since the independent variable $t$ does not explicitly appear in Eq. (9), the problem has the energy as a first integral

$$
H\left(\frac{d r}{d t}, r\right)=\frac{1}{2}\left(\left(\frac{d r}{d t}\right)^{2}+\frac{\Theta^{2}}{r^{2}}\right)-\frac{\mu}{r}+\varepsilon \frac{\Phi}{r^{3}}=H\left(\frac{d r}{d t}\left(t_{0}\right), r\left(t_{0}\right)\right)=\widetilde{h}
$$

for all $t_{0}$ and $t$.

Next we introduce in Eq. (9) a new independent variable $\theta=\theta(t)$ along each solution $r=r(t)$ defined by

$$
\frac{d \theta}{d t}=\frac{\Theta}{r(t)^{2}}, \quad \theta(0)=0
$$

so that

$$
\theta(t)=\int_{0}^{t} \frac{\Theta}{r(s)^{2}} d s
$$

is a monotonic increasing function of $t$.

Denoting by $u=u(\theta)$ the function of the new independent variable $\theta$ such that

$$
u(\theta(t))=\frac{1}{r(t)}
$$

we have

$$
\frac{d u}{d \theta}=-\frac{1}{\Theta} \frac{d r}{d t}, \quad \frac{d^{2} u}{d \theta^{2}}=-\frac{r^{2}}{\Theta^{2}} \frac{d^{2} r}{d t^{2}} .
$$

Hence in view of $(9)$ we get

$$
\frac{d^{2} u}{d \theta^{2}}=-u+\frac{\mu}{\Theta^{2}}-\varepsilon \frac{3 \Phi}{\Theta^{2}} u^{2},
$$

that is the differential equation of a non linear harmonic oscillator. 


\subsection{Solution of Cid's intermediary by elliptic functions}

We consider the general case in which $0 \leq|\varepsilon| \ll 1$. To give an explicit form of the solution of $12 p$ observe that after multiplication by $2(d u / d \theta)$ we have the first integral

$$
\left(\frac{d u}{d \theta}\right)^{2}=-u^{2}+\frac{2 \mu}{\Theta^{2}} u-\varepsilon \frac{2 \Phi}{\Theta^{2}} u^{3}+K_{0}
$$

where $K_{0}$ is a constant of integration related to the energy $10 p: \widetilde{h}=H\left(R_{0} ; r_{0}\right)$ by

$$
K_{0}=\frac{2 \widetilde{h}}{\Theta^{2}}
$$

Then, we have

$$
\left(\frac{d u}{d \theta}\right)^{2}=F(u) \equiv-u^{2}+\frac{2 \mu}{\Theta^{2}} u-\varepsilon \frac{2 \Phi}{\Theta^{2}} u^{3}+\frac{2 \widetilde{h}}{\Theta^{2}},
$$

Next following the ideas of the unperturbed case $\varepsilon=0$ (see e.g. [16]) we introduce instead of $\widetilde{h}$ and $\Theta$ the alternative constants

$$
\widetilde{a}=-\frac{\mu}{2 \widetilde{h}}, \quad \frac{1}{p}=\frac{\mu}{\Theta^{2}}, \quad \widetilde{e}=\left(1+\frac{2 \widetilde{h} \Theta^{2}}{\mu^{2}}\right)^{1 / 2}
$$

that satisfy

$$
p=\widetilde{a}\left(1-\widetilde{e}^{2}\right) .
$$

We also assume that the initial conditions satisfy $\widetilde{h}<0$ and $0 \leq \widetilde{e}<1$.

Note that $\widetilde{a}$ and $\widetilde{e}$ reduce to the semimajor axis and eccentricity respectively in the case $\varepsilon=0$ but now the orbit is no longer a conic and does not make sense to use these names.

With the notations 15, 16) the function $F(u)$ of Eq. 14 becomes

$$
F(u)=-u^{2}+\frac{2}{p} u-\varepsilon \frac{2 \Phi p}{\mu} u^{3}-\frac{1-\widetilde{e}^{2}}{p^{2}},
$$

Let us introduce the change

$$
v=p u, \quad \text { and } \quad \widetilde{\varepsilon}=-\varepsilon \frac{2 \Phi}{\mu},
$$

then

$$
F(u)=-u^{2}+\frac{2}{p} u+\widetilde{\varepsilon} p u^{3}-\frac{1-\widetilde{e}^{2}}{p^{2}},
$$


and the return points are the roots of the cubic equation $F(u)=0$, or equivalently the roots of

$$
\widetilde{F}(v)=p^{2} F(u)=\widetilde{\varepsilon} v^{3}-v^{2}+2 v-\left(1-\widetilde{e}^{2}\right) .
$$

The discriminant $\Delta$ of the cubic polynomial $\widetilde{F}(x)$ is

$$
\Delta=4 \widetilde{e}^{2}+4\left(1-9 \widetilde{e}^{2}\right) \widetilde{\varepsilon}-27\left(1-\widetilde{e}^{2}\right)^{2} \widetilde{\varepsilon}^{2},
$$

and $\widetilde{F}(x)$ has three real and distinct real roots if and only if their coefficients satisfy $\Delta>0$. Next, we will assume that the initial conditions in addition of $H_{0}<0$ satisfy this condition. Note that the case of $\widetilde{F}(x)$ with a double root $(\Delta=0)$, i.e., circular solutions could be also studied, although this case is not considered here.

In this setting the cubic equation $\widetilde{F}(v)=0$ has three real roots, let us say $v_{s}, v_{p}$, and $v_{a}$, such that when $\widetilde{\varepsilon} \rightarrow 0$

$$
v_{s} \rightarrow \pm \infty, \quad v_{p} \rightarrow 1+\widetilde{e}, \quad v_{a} \rightarrow 1-\widetilde{e} .
$$

We do not include here an explicit expression of these roots that can be obtained with an algebraic manipulator, e.g. with Mathematica, because it is quite complicated, but the first order terms of their series expansions in powers of $\widetilde{\varepsilon}$ are

$$
\begin{aligned}
& v_{s}=\frac{1}{\widetilde{\varepsilon}}-2-\left(3+\widetilde{e}^{2}\right) \widetilde{\varepsilon}-2\left(5+3 \widetilde{e}^{2}\right) \widetilde{\varepsilon}^{2}+\mathcal{O}\left(\widetilde{\varepsilon}^{3}\right), \\
& v_{a}=(1-\widetilde{e})-\frac{(1-\widetilde{e})^{3}}{2 \widetilde{e}} \widetilde{\varepsilon}+\frac{(1-\widetilde{e})^{5}(1+5 \widetilde{e})}{8 \widetilde{e}^{3}} \widetilde{\varepsilon}^{2}+\mathcal{O}\left(\widetilde{\varepsilon}^{3}\right)
\end{aligned}
$$

and $v_{p}$ is obtained from $v_{a}$ substituting $\widetilde{e}$ by $-\widetilde{e}$.

Now in the $(u=p v)$-variable, they correspond to the values

$$
u_{s}=p v_{s}, \quad u_{p}=p v_{p}, \quad u_{a}=p v_{a} .
$$

And the oscillatory motions occur for $u$ between $u_{a}$ and $u_{p}$ so that the angle $T_{\theta}$ to go from $u_{a} \rightarrow u_{p} \rightarrow u_{a}$ is

$$
T_{\theta}=2 \int_{u_{a}}^{u_{p}} \frac{d u}{\sqrt{F(u)}}
$$


with $F(u)$ given by Eq. 18$)$. Note that if $T_{\theta}$ is a rational multiple of $2 \pi$ the orbit is closed and the motion is periodic.

Note that $v_{s}$ is the spurious root in the sense that appears when the leading order polynomial. The other two roots $v_{p}$ and $v_{a}$, because $v=p u=p / r$ tend to the corresponding values at perigee and apogee respectively.

Now since $F(u)>0$ for $u \in\left[u_{a}, u_{p}\right]$, the equation

$$
\left(\frac{d u}{d \theta}\right)^{2}=F(u)
$$

defines $\theta$ as a function of $u$ by the integral

$$
\theta=\int_{u_{a}}^{u} F(\xi)^{-1 / 2} d \xi, \quad \text { for } \quad u \in\left[u_{a}, u_{p}\right]
$$

To give an explicit expression of the inverse function $u=u(\theta)$ of the above Eq. 22 observe that the elliptic Weierstrass function $\mathcal{P}=\mathcal{P}\left(z ; w_{1}, w_{2}\right)$ with periods $w_{1}, w_{2}$ (see [29, Chapter XX, page 429]) satisfies the differential equation

$$
\left(\frac{d \mathcal{P}}{d z}\right)^{2}=4 \mathcal{P}^{3}-g_{2} \mathcal{P}-g_{3}
$$

where $g_{2}, g_{3}$ are the so called invariants that depend on $w_{1}, w_{2}$. Since Eq. 13 after a trivial change of variable $u \rightarrow \alpha_{1} u+\alpha_{0}$ can be written in the form 23 , it is clear that the solution of 22 can be written explicitly in terms of $\mathcal{P}$ functions.

In fact, an explicit expression can be given by using section 20.6 of [29], pages 452-453 which proves:

Proposition 1. Let

$$
a_{0} t^{4}+4 a_{1} t^{3}+6 a_{2} t^{2}+4 a_{3} t+a_{4}=G(t)
$$

be any quartic polynomial which has no repeated factors. Let its invariants be

$$
\begin{aligned}
& g_{2}=a_{0} a_{4}-4 a_{1} a_{3}+3 a_{2}^{2}, \\
& g_{3}=a_{0} a_{2} a_{4}+2 a_{1} a_{2} a_{3}-a_{2}^{3}-a_{0} a_{3}^{2}-a_{1} a_{4} .
\end{aligned}
$$


If $G(a)=0$ then

$$
z=\int_{a}^{x} G(t)^{-1 / 2} d t
$$

defines the function $x=x(z)$ by

$$
x=a+\frac{(1 / 4) G^{\prime}(a)}{\mathcal{P}\left(z ; g_{2}, g_{3}\right)-(1 / 24) G^{\prime \prime}(a)} .
$$

Now since from 21 $(d u / d \theta)^{2}=F(u)$ and the relations $v=p u$, and $\widetilde{F}(v)=$ $p^{2} F(u)$, we have

$$
\left(\frac{d v}{d \theta}\right)^{2}=\widetilde{F}(v)=\widetilde{\varepsilon} v^{3}-v^{2}+2 v-\left(1-\widetilde{\varepsilon}^{2}\right)
$$

Then we apply Eq. 24, with

$$
a=v_{a}, \quad x=v, \quad G=\widetilde{F},
$$

i.e.,

$$
a_{0}=0, \quad a_{1}=\widetilde{\varepsilon} / 4, \quad a_{2}-1 / 6, \quad a_{3}=1 / 2, \quad a_{4}=-\left(1-\widetilde{e}^{2}\right),
$$

obtaining

$$
u=\frac{1}{p}\left(v_{a}+\frac{(1 / 4) \widetilde{F}^{\prime}\left(v_{a}\right)}{\mathcal{P}\left(\theta ; g_{2}, g_{3}\right)-(1 / 24) \widetilde{F}^{\prime \prime}\left(v_{a}\right)}\right),
$$

with

$$
\begin{array}{ll}
g_{2}=\frac{1}{12}(1-6 \tilde{e}), & g_{3}=\frac{1}{216}\left(1+35 \tilde{e}-54 \tilde{e}^{3}\right), \\
\widetilde{F}^{\prime}\left(v_{a}\right)=2-2 a+3 \tilde{\varepsilon} a^{2}, & \widetilde{F}^{\prime \prime}\left(v_{a}\right)=2(-1+3 \tilde{\varepsilon} a),
\end{array}
$$

that is the explicit equation of the orbit $u=u(\theta)$ where $u=1 / r$, the solution of 14 in terms of the elliptic Weierstrass function $\mathcal{P}\left(\theta ; g_{2}, g_{3}\right)$.

Incidentally, let us mention that another analytic expression of the orbit can be given by using elliptic Jacobian functions. To this task we recall some results of elliptic functions.

Proposition 2. Integrals of cubic radicals.

For solving the integral

$$
I(y)=\int_{c}^{y} \frac{d z}{\sqrt{(a-z)(b-z)(z-c)}}
$$


where $a, b$, an $c$ are real constants with $a>b \geq y>c$, in [8, §233, $p$. 72] the authors propose the following change of variable $z \rightarrow \xi$ defined by

$$
\operatorname{sn}^{2}(\xi ; k)=\frac{z-c}{b-c}, \quad k^{2}=\frac{b-c}{a-c},
$$

i.e.,

$$
z=c+(b-c) \operatorname{sn}^{2}(\xi ; k)
$$

Then

$$
I(y)=g w_{1}=g \operatorname{sn}^{-1}(\sin \psi ; k)=g F(\psi ; k),
$$

where

$$
g=\frac{2}{\sqrt{a-c}}, \quad \psi=\operatorname{am}\left(w_{1}\right)=\arcsin \left(\sqrt{\frac{y-c}{b-c}}\right), \quad \operatorname{sn} w_{1}=\sin \psi
$$

Then since by 14

$$
\theta=\int_{u_{p}}^{u} \frac{d u}{\sqrt{F(u)}}=\int_{v_{p}}^{v} \frac{d v}{\sqrt{\widetilde{F}(v)}},
$$
in obtaining this goal is by means of the Sundman regularizing transformation $d t=r d E$, where $E$ is the eccentric anomaly [28, p. 127]. In our case, we 
shall proceed in a different way, first changing the radial distance, and second, introducing as independent variable the polar angle $\theta$.

In this context, we introduce along a solution $r=r(t)$ of 9 a new independent variable $\tau=\tau(t)$ defined by

$$
\frac{d \tau}{d t}=\frac{1}{r(t)}>0, \quad \tau(0)=0,
$$

i.e., $\tau$ is a monotonic function of $t$ given by

$$
\tau=\tau(t)=\int_{0}^{t} \frac{d s}{r(s)}
$$

By the chain rule, the derivatives of $r(t)$ can be written as

$$
\frac{d r}{d t}=\frac{1}{r} \frac{d r}{d \tau}, \quad \frac{d^{2} r}{d t^{2}}=\frac{1}{r^{2}} \frac{d^{2} r}{d \tau^{2}}-\frac{1}{r}\left(\frac{d r}{d t}\right)^{2},
$$

Putting $\rho=\rho(\tau)$ so that $\rho(\tau(t))=r(t)$ we have

$$
\frac{d^{2} r}{d t^{2}}=\frac{1}{\rho^{2}} \frac{d^{2} \rho}{d \tau^{2}}-\frac{1}{r}\left(\frac{d r}{d t}\right)^{2}
$$

and substituting into $(9)$ and taking into account the energy integral 10 we have

$$
\frac{d^{2} \rho}{d \tau^{2}}=\mu+2 \widetilde{h} \rho+\varepsilon \frac{\Phi}{\rho^{2}} .
$$

Therefore, the differential equation satisfied by $\rho=\rho(\tau)$ is a perturbed harmonic oscillator. Hereafter we will assume that the energy $\widetilde{h}<0$ and then for $\varepsilon=0$ the orbit is $\tau$-periodic with frequency $w=\sqrt{-2 \widetilde{h}}$.

To approximate the solution of Eq. (26) we use Krylov-Bogoliubov averaging method [18] that we recall here. For details on this averaging method, see e.g. [1.

Proposition 3. The KB averaging method.

Suppose that $w>0$ and $\mu>0$ are given constants and $0 \leq \epsilon \ll w$ a small parameter. Let $\rho=\rho(\tau ; \epsilon)$ be the solution of

$$
\left\{\begin{array}{l}
\frac{d^{2} \rho}{d \tau^{2}}+w^{2} \rho=\mu+\epsilon f(\rho), \\
\rho(0)=\rho_{0}, \quad \frac{d \rho}{d \tau}(0)=\rho_{0}^{\prime}
\end{array}\right.
$$


with $f(\rho)$ a sufficiently smooth function.

Let $\rho(\tau ; 0)$ be the solution of (27) corresponding to $\epsilon=0$ that is an harmonic oscillator and can be written in the form

$$
\rho(\tau ; 0)=\frac{\mu}{w^{2}}+A_{0} \cos \left(w \tau+B_{0}\right)
$$

with $A_{0}$ and $B_{0}$ constants of integration given by the initial conditions

$$
\frac{\mu}{w^{2}}+A_{0} \cos B_{0}=\rho_{0}, \quad-A_{0} w \sin B_{0}=\rho_{0}^{\prime},
$$

or equivalently

$$
A_{0}= \pm \sqrt{\left(\rho_{0}-\frac{\mu}{w^{2}}\right)^{2}+\frac{\rho_{0}^{\prime 2}}{w^{2}}}, \quad \tan B_{0}=\frac{\rho_{0}^{\prime} w}{\mu-\rho_{0} w^{2}} .
$$

Then, the so called averaged solution of 27) is given by

$$
\widetilde{\rho}(\tau ; \epsilon)=\frac{\mu}{w^{2}}+\widetilde{A} \cos (w \tau+\widetilde{B})
$$

with

$$
\widetilde{A}=\widetilde{A}(\tau, \epsilon), \quad \widetilde{B}=\widetilde{B}(\tau, \epsilon)
$$

satisfying the differential equations

$$
\begin{aligned}
& \frac{d \widetilde{A}}{d \tau}=-\left(\frac{\epsilon}{2 \pi w}\right) \int_{0}^{2 \pi} \sin \varphi f\left(\mu / w^{2}+\widetilde{A} \cos \varphi\right) d \varphi, \\
& \frac{d \widetilde{B}}{d \tau}=-\left(\frac{\epsilon}{2 \pi w \widetilde{A}}\right) \int_{0}^{2 \pi} \cos \varphi f\left(\mu / w^{2}+\widetilde{A} \cos \varphi\right) d \varphi,
\end{aligned}
$$

where now $\widetilde{A}$ is held fixed during the integration in the right hand side of (30).

It has been proved that the averaged solution $\widetilde{\rho}(\tau ; \epsilon)$ defined by $(28)$ and (30) satisfies

$$
|\rho(\tau ; \epsilon)-\widetilde{\rho}(\tau ; \epsilon)| \leq C_{1} \epsilon
$$

for $\tau \in\left[0, C_{2} / \epsilon\right]$ for some constants $C_{1}, C_{2}$ independent of $\epsilon$.

Let us apply this averaging method to Eq. 26). In our case $f(y)=1 / y^{2}$ and, firstly, for $|\nu|<1$, we compute the quadratures

$$
\frac{1}{2 \pi} \int_{0}^{2 \pi} \frac{\sin \varphi}{(1+\nu \cos \varphi)^{2}} d \varphi=0, \text { and } \frac{1}{2 \pi} \int_{0}^{2 \pi} \frac{\cos \varphi}{(1+\nu \cos \varphi)^{2}} d \varphi=\Psi(\nu),
$$


with

$$
\Psi(\nu)=-\frac{\nu}{\left(1-\nu^{2}\right)^{(3 / 2)}} .
$$

Taking into account these values of the quadratures, and putting

$$
\nu=\frac{\widetilde{A} w^{2}}{\mu},
$$

where we assume $|\nu|<1$, for the first equation of $(30)$, we have

$$
\frac{d \widetilde{A}}{d \tau}=-\frac{\epsilon}{2 \pi w} \frac{w^{4}}{\mu^{2}} \int_{0}^{2 \pi} \frac{\sin \varphi}{(1+\nu \cos \varphi)^{2}} d \varphi=0 ;
$$

then, $\widetilde{A}=\widetilde{A}(0)=\widetilde{A}_{0}$ (constant).

For the second equation of $(30)$, there results (see Appendix A)

$$
\frac{d \widetilde{B}}{d \tau}=-\frac{\epsilon}{2 \pi w \widetilde{A}} \frac{w^{4}}{\mu^{2}} \int_{0}^{2 \pi} \frac{\cos \varphi}{(1+\nu \cos \varphi)^{2}} d \varphi=-\frac{\epsilon w^{3}}{\widetilde{A} \mu^{2}} \Psi(\nu),
$$

and therefore, if $\widetilde{B}(0)=\widetilde{B}_{0}$,

$$
\widetilde{B}(\tau, \epsilon)=\widetilde{B}_{0}-\frac{\epsilon w^{3}}{\widetilde{A} \mu^{2}} \Psi(\nu) \tau .
$$

In conclusion, the averaged solution is

$$
\widetilde{\rho}(\tau ; \epsilon)=\frac{\mu}{w^{2}}+\widetilde{A}_{0} \cos \left(\widetilde{w} \tau+\widetilde{B}_{0}\right)
$$

with

$$
\widetilde{w}=w-\frac{\epsilon w^{3}}{\widetilde{A}_{0} \mu^{2}} \Psi(\nu)
$$

Next, to obtain the relation between the regularizing variable $\tau$ and the physical time $t$, observe that $\rho d \tau=d t$ can be written as

$$
\left(\frac{\mu}{w^{2}}+\widetilde{A}_{0} \cos \left(\widetilde{w} \tau+\widetilde{B}_{0}\right)\right) d \tau=d t,
$$

and integrating both sides

$$
\left(\frac{\mu}{w^{2}}\right) \tau+\frac{\widetilde{A}_{0}}{\widetilde{w}}\left(\sin \left(\widetilde{w} \tau+\widetilde{B}_{0}\right)-\sin \widetilde{B}_{0}\right)=t .
$$

Introducing instead of $\tau$ the new variable

$$
\widetilde{E}=\widetilde{w} \tau+\widetilde{B}_{0},
$$


and the constants

$$
\widetilde{e}=-\frac{\widetilde{A}_{0} w^{2}}{\mu}, \quad \widetilde{n}=\frac{w^{2} \widetilde{w}}{\mu}, \quad \widetilde{t}=-\frac{\mu}{w^{2} \widetilde{w}} \widetilde{B}_{0}-\frac{\widetilde{A}_{0}}{\widetilde{w}} \sin \widetilde{B}_{0},
$$

equation (34) becomes

$$
\widetilde{E}-\widetilde{e} \sin \widetilde{E}=\widetilde{n}(t-\widetilde{t})
$$

that for each physical time value $t$ defines $\widetilde{E}$ and consequently $\tau=\left(\widetilde{E}-\widetilde{B}_{0}\right) / \widetilde{w}$ that gives the corresponding radius vector.

Note that assuming $0 \leq \widetilde{e}<1$ this is a Kepler's type equation that can be solved by means of the standard solvers.

Taking into account the $r^{2} d \theta / d \tau=\Theta$ and proceeding as in the Kepler's problem to relate the true and the eccentric anomaly, it can be seen that

$$
\tan (\theta / 2)=\sqrt{\frac{1-\widetilde{e}}{1-\widetilde{e}}} \tan (\widetilde{E} / 2) .
$$

Finally, let us remark that due to the simplicity of the KB averaged solution the calculation of both position and velocity at any physical time is computationally equivalent to the same calculation in a pure Kepler's problem. In particular for each time one has to solve a Kepler's equation for which there exist a well developed collection of methods with good accuracy and efficiency. Furthermore, since the exact solution of $26, r=\rho(\tau, \epsilon)$ and the averaged solution $r=\widetilde{\rho}(\tau, \epsilon)$ satisfy

$$
|\rho(\tau, \epsilon)-\widetilde{\rho}(\tau, \epsilon)| \leq C_{1} \epsilon
$$

for $\tau \in\left[0, C_{2} / \epsilon\right)$ with constants $C_{1}, C_{2}$ independent of $\epsilon$, the same bounds (although with different constants) hold for the radius vectors as functions of the "eccentric" anomaly $(\widetilde{E})$ and the physical time $(t)$, in intervals of lengths $C_{2} / \epsilon$.

In conclusion, the averaged solution provides a first order approximation to the exact solution that holds true in time intervals of size $1 / \epsilon$ and this accuracy may be sufficient for many applications like mission analysis or orbit design, where fast evaluation is preferred to high accuracy and where long time propagation is required (see e.g. [2]). 


\section{Conclusions}

We present two solutions of the Cid's radial intermediary problem: An exact solution of the polar equation of the orbit $r=r(\theta)$ in which $1 / r$ is expressed in terms of Weierstrass $\mathcal{P}\left(\theta ; g_{2}, g_{3}\right)$ functions where the invariants $g_{2}, g_{3}$ depend on the eccentricity $e$. An alternative expression of the exact solution is also given

\section{References}

[1] A. Abad, M. Calvo, A. Elipe, Integration of Deprit's ra-

An approximate solution is derived by using the KB-averaging method. We showed that Cid's radial intermediary can be reduced to a pure Kepler-type problem, and hence, there is no need to introduce generalized Kepler equations, because the time position on the orbit can be obtained from the classical Kepler's equation.

KB-averaging method provides error bounds for the solution in time intervals of size $1 / \epsilon$, and it is quite convenient for studies which require long time intervals because its complexity is similar to use a pure Kepler's problem.

\section{Acknowledgments}

Authors are very indebted to the two anonymous reviewers whose comments and suggestions improved the manuscript. This work has been supported by the Spanish Ministry of Economy (projects ESP2017-87113-R and MTM2016-77735-C3-1-P) and by the Aragon Government and European Social Fund (groups E24_20R and E41_20R ).

The authors declare that there are no conflicts of interest regarding the publication of this paper.

https://doi.org/10.1016/j.actaastro.2020.03.039.

dial intermediary. Acta Astronautica $173 \quad$ (2020) 19-21,
https://doi.org/10.1016/j.actaastro.2020.03.039.


[2] A. Abad, A. Elipe, J.F. San-Juan, S. Serrano, Is symbolic integration better tham numerical integration in Satellite dynamics?. Ap. Math. L. 17 (2004) 59-63, https://doi.org/10.1016/S0893-9659(03)00219-2

[3] A. Abad, A. Gavín, A., J.F. San-Juan, Short term evolution of artificial satellites. Celest. Mech. Dyn. Astron. 79 (2001) 277-296, https://doi.org/10.1023/A:1017540603450.

[4] K.T. Alfriend, S.L. Coffey, Elimination of the perigee in satellite problem. Celest. Mech. Dyn. Astron. 32 (1984) 163-172.

[5] M. Arribas, A. Elipe, M. Palacios, Linear stability of ring systems with generalized central forces. Astron. Astroph. 489 (2008) 819-824, https://doi.org/10.1051/0004-6361:200809903

[6] M. Arribas, A. Elipe, M. Palacios, T. Kalvouridis, Homographic solutions in the planar $n+1$ body problem with quasi-homogeneous potentials. Celest. Mech. Dyn. Astron. 99 (2007) 1-12, https://doi.org/10.1007/s10569-0079083-8

[7] M. Arribas, A. Elipe, A. Riaguas, Non-integrability of anisotropic quasi homogeneous Hamiltonian systems, Mech. Res. Comm. 30 (2003) 209-216, https://doi.org/10.1016/S0093-6413(03)00005-3

[8] P.F. Byrd and M.D. Friedman, Handbook of Elliptic Integrals for Engineers and Scientists, Second Revised Edition, Springer Verlag, Berlin (1971), ISBN: 978-3-642-65140-3. https://doi.org/10.1007/978-3-642-65138-0

[9] J.A.Caballero, S. Ferrer, M.L. Sein-Echaluce, Second order solution of a radial intermediary in satellite theory. In Space Dynamics and Celestial Mechanics. Proceedings of the International Workshop, Delhi, India, Nov. 14-16, 1985. Edited by K. B. Bhatnagar. Dordrecht, D. Reidel Publishing Co. Astrophysics and Space Science Library. Vol. 127 (1986) 243-253, https://doi.org/10.1007/978-94-009-4732-0_23. 
[10] R. Cid, S. Ferrer, A. Elipe, Regularization and linearization of the equations of motion in central force-fields. Celest. Mech. 31 (1983) 73-80, https://doi.org/10.1007/BF01272561

[11] R. Cid, S. Ferrer, M.L. Sein-Echaluce, On the radial intermediaries and the time transformation in satellite theory. Celest. Mech. 38 (1986) 191-205, https://doi.org/10.1007/BF01230431

[12] R. Cid, J.F. Lahulla, Perturbaciones de corto periodo en el movimiento de un satélite artificial, en función de las variables de Hill. Rev. Acad. Ciencias Zaragoza 24 (1969) 159-165.

[13] A. Deprit, The elimination of the parallax in satellite theory. Celest. Mech. 24 (1981) 111-153, https://doi.org/10.1007/BF01229192.

[14] A. Deprit, B. Miller, Simplify or Perish. Celest. Mech. 45 (1989) 189-200

[15] J.M. Ferrándiz, Linearization in special cases of perturbed Keplerian motions. Celest. Mech. 39 (1986) 23-31, https://doi.org/10.1007/BF01232286

[16] H. Goldstein, Classical Mechanics, (2nd Edition). Addison Wesley Pub., N.Y. (1980) ISBN: 978-0-201-65702-9

[17] Y. Hagihara, Celestial Mechanics, vol. 2, part I, MIT Press, Cambridge, Mass. (1975)

[18] N.M. Krylov, N.N. Bogoliubov, Introduction to Nonlinear Mechanics, Princeton University Press, Princeton N.Y. (1947), ISBN: 9780691079851.

[19] M. Lara, A new radial, natural, higher-order intermediary of the main problem four decades after the elimination of the parallax. Celest. Mech. Dyn. Astron. 131:42 (2019), https://doi.org/10.1007/s10569-019-9921-5

[20] M. Lara, P. Gurfil, Integrable approximation of $J_{2}$-perturbed relative orbits. Celest. Mech. Dyn. Astron. 114 (2012) 229-254, https://doi.org/10.1007/s10569-012-9437-8 
[21] J. Llibre, A.E. Teruel, C. Valls, A. de la Fuente, Phase portraits of the two-body problem with Manev potential, J. Phys. A: Math. Gen. 34 (2001) 1919-1934, https://doi.org/10.1088/0305-4470/34/9/309

[22] R. López, D. Hautesserres, J.F. San-Juan, The solution of the Generalized Kepler's equation. MNRAS 473 (2018) 2583-2589, https://doi.org/10.1093/mnras/stx2522.

[23] G. Maneff, La gravitation et le principe de l'égalité de l'action et de la réaction, Comptes Rendus 178 (1924) 2159-2161.

[24] G. Maneff, Le principe de la moindre action et la gravitation Comptes Rendus 190 (1930) 963-965

[25] V. Mioc, C. Stoica, Discussion et résolution complète du problème des deux corps dans le champ gravitationnel de Maneff, C. R. Acad. Sci. Paris 320 (1995) 645-648.

[26] V. Mioc, M. Stavinschi, On the Schwarzschild-type polygonal $(n+1)$-body problem and on the associated restricted problem, Baltic Astron. 7 (1998) 637-651, https://doi.org/10.1515/astro-1998-0408

[27] I. Newton, Isaac Newton's PhilosophiæNaturalis Principia Mathematica. The Third Edition (1726). Assembled by A. Koyré and B.Cohen, Harvard Univ. Press. Cambridge, Mass. (1972)

[28] K.F. Sundman, Mémoire sur le problème des trois corps. Acta Math. 36 300 (1913) 105-179, https://doi.org/10.1007/BF02422379

[29] E.T. Whittaker, G.N. Watson, A course in Modern Analysis, fourth edition, Cambridge University Press, Cambridge (1950), https://doi.org/10.1002/zamm.19630430916 


\section{Appendix A. The computation of $\Psi=\Psi(\nu)$}

First of all observe that the function $g_{1}(x ; \nu)$ defined by the integral

$$
g_{1}(x ; \nu)=\int \frac{d x}{(1+\nu \cos x)} \quad \text { with } \quad|\nu|<1
$$

satisfies that

$$
g_{2}(x ; \nu)=-\frac{\partial g_{1}(x ; \nu)}{\partial \nu}=\int \frac{\cos x d x}{(1+\nu \cos x)^{2}}
$$

which is the integral that appears in $\Psi(\nu)$ (see Eq. (30)).

Now it can be easily obtained that

$$
g_{1}(x ; \nu)=\frac{2}{\sqrt{1-\nu^{2}}} \arctan \left(\sqrt{\frac{1-\nu}{1+\nu}} \tan (x / 2)\right)
$$

and then from A.1 to obtain the explicit expression of $g_{2}$

$$
g_{2}(x ; \nu)=\frac{-1}{1-\nu^{2}}\left[\nu g_{1}(x ; \nu)-\frac{\sin x}{(1+\nu \cos x)}\right] \text {. }
$$

But the point here is that both functions $g_{1}(x ; \nu)$ and $g_{2}(x ; \nu)$ are discontinuous of $x$ in the interval $[0,2 \pi]$ at $x=\pi$. Hence Barrow's rule cannot be applied in the whole interval and then we should decompose in the two subintervals

$$
\int_{0}^{2 \pi} \frac{d x}{1+\nu \cos x}=\int_{0}^{\pi} \frac{d x}{1+\nu \cos x}+\int_{\pi}^{2 \pi} \frac{d x}{1+\nu \cos x},
$$

But because the $1 /(1+\nu \cos x)$ is an even and $2 \pi$-periodic function,

$$
\int_{0}^{2 \pi} \frac{d x}{1+\nu \cos x}=2 \int_{0}^{\pi} \frac{d x}{1+\nu \cos x}=2\left[g_{1}\left(\pi^{-} ; \nu\right)-g_{1}(0 ; \nu)\right]
$$

with

$$
g_{1}\left(\pi^{-} ; \nu\right)=\lim _{x \rightarrow \pi, x<\pi} g_{1}(x ; \nu)=\frac{\pi}{\sqrt{1-\nu^{2}}}, \quad g_{1}(0 ; \nu)=0,
$$

then

$$
\int_{0}^{2 \pi} \frac{d x}{1+\nu \cos x}=\frac{2 \pi}{\sqrt{1-\nu^{2}}}
$$

By the same token, we obtain that

$$
\int_{0}^{2 \pi} \frac{\cos x d x}{(1+\nu \cos x)^{2}}=2\left[g_{2}\left(\pi^{-} ; \nu\right)-g_{2}(0 ; \nu)\right]=\frac{-2 \pi \nu}{\left(1-\nu^{2}\right)^{3 / 2}} .
$$

that leads easily to the result in Eq. 32. 